\title{
Neurochemical Afferents Controlling the Activity of Serotonergic Neurons in the Dorsal Raphe Nucleus: Microiontophoretic Studies in the Awake Cat
}

\author{
Eric S. Levine and Barry L. Jacobs \\ Program in Neuroscience, Department of Psychology, Princeton University, Princeton, New Jersey 08544
}

Serotonergic (5-HT) neurons of the brainstem dorsal raphe nucleus (DRN) have been implicated in a diversity of physiological and behavioral processes in vertebrates. However, despite extensive information about the intrinsic properties and the efferent projections of this neurochemical system, little information is available regarding the afferents that control its activity. This study investigated the neurotransmitters that regulate the activity of DRN-5-HT neurons under physiologically relevant conditions, by utilizing microiontophoresis in combination with single-unit recordings in the awake, head-restrained cat. This made it possible to examine the direct effects of neurotransmitters on DRN-5-HT neuronal activity, and, through the use of specific antagonists, to study the roles of these neurotransmitter inputs during physiological conditions that influence DRN-5-HT neuronal activity.

The results indicate that (1) iontophoretic application of the GABA antagonist bicuculline reversed the typical suppression of neuronal activity seen during slow wave sleep, but had no effect on maintained activity during wakefulness. The suppression of neuronal activity during REM sleep was generally unaffected by application of bicuculline. This suggests a role for a GABAergic input to DRN-5-HT neurons in controlling some aspects of their state-dependent activity. (2) Iontophoretic application of the excitatory amino acid (EAA) antagonist kynurenic acid reduced the magnitude of the neuronal response evoked by phasic auditory stimuli, but had no effect on the spontaneous activity of these neurons, suggesting a role for an EAA input to the DRN in mediating the response to phasic sensory stimuli. (3) Iontophoretic application of norepinephrine or the $\alpha$-adrenergic agonist phenylephrine did not alter spontaneous neuronal activity during waking, suggesting that the noradrenergic input to these neurons already exerts a maximal effect during wakefulness.

These studies demonstrate the utility of microiontophoresis in the awake animal in delineating the functional roles of specific neurotransmitter inputs to DRN-5-HT neurons.

\footnotetext{
Received Feb. 13, 1992; revised May 14, 1992; accepted May 27, 1992.

This work was supported by grants from the Air Force Office of Scientific Research (90-0294) and the National Institute of Mental Health (MH 23433) to B.L.J., and a National Science Foundation predoctoral fellowship to E.S.L. We gratefully acknowledge the assistance of Dr. Robert Holdefer and Dr. David Morilak in the initial stages of this work. We also thank Ms. Christinc Mctzlcr for excellent technical assistance.

Correspondence should be addressed to Eric S. Levine, Ph.D., Department of Neuroscience and Cell Biology, UMDNJ-Robert Wood Johnson Medical School, 675 Hoes Lane, Piscataway, NJ 08854.

Copyright (C) 1992 Society for Neuroscience $0270-6474 / 92 / 124037-08 \$ 05.00 / 0$
}

This information provides an important link in understanding the role of the DRN-5-HT system in physiological and behavioral processes.

Serotonergic (5-HT) neurons of the dorsal raphe nucleus (DRN) have been implicated in a range of behavioral and physiological processes, including feeding, nociception, motor control, sleep, and thermoregulation (Whitaker-Azmitia and Peroutka, 1990). However, despite extensive information about the intrinsic properties and efferent projections of this system (Jacobs and Azmitia, 1992), the afferent control of DRN-5-HT neuronal activity is not well understood. It is not known, for example, which neurochemical inputs are responsible for producing the decrease in 5-HT neuronal activity during sleep (McGinty and Harper, 1976; Trulson and Jacobs, 1979), or the phasic increase in activity in response to sensory stimuli (Heym et al., 1982; Shima et al., 1986). The present experiments represent the first attempt to study this issue by utilizing microiontophorcsis in combination with extracellular single-unit recordings in the awake cat. These studies attempted to identify the inputs that regulate the activity of these neurons under physiological conditions. This was done by using antagonists to inactivate specific neurochemical inputs during the conditions that influence the activity of these neurons.

The DRN receives input from many anatomical areas that utilize a diversity of neurotransmitters, with major inputs originating in the lateral habenula, hypothalamus, pontine reticular formation, and other raphe nuclei (Aghajanian and Wang, 1977; Sakai et al., 1977; Jacobs and Azmitia, 1992). Among the neurotransmitters present in these projections is a prominent GABAergic input. A high density of GABAergic terminals and fibers is found in the DRN (Belin et al., 1979), as well as high levels of GABA and its synthesizing enzyme glutamic acid decarboxylase (Gottesfeld et al., 1978; Belin et al., 1979; Vincent ct al., 1980). In studies performed in anesthetized rats, iontophoretically applied GABA inhibits the activity of DRN-5-HT neurons and this inhibition is reversed by specific GABA antagonists (Gallager and Aghajanian, 1976b; Lakoski and Aghajanian, 1983; VanderMaelen et al., 1986). In addition, local infusion of GABA into the DRN decreases 5-HT synthesis and metabolism in the corpus striatum (Nishikawa and Scatton, 1982; Scatton el al., 1984). Some attempts have been made to identify the afferents that provide the GABAergic input to the DRN. Electrical stimulation of both the lateral habenula and the pontine reticular formation inhibits DRN-5-HT neuronal activity, and this inhibition is reversed by GABA antagonists (Wang et al., 1976; Wang and Aghajanian, 1977; Stern et al., 1981), although these 
effects may be mediated by GABAergic interneurons located within the DRN (Belin et al., 1979). The functional roles, however, of these GABAergic inputs are unclear.

There is also evidence for excitatory amino acid (EAA) inputs to the DRN. Microiontophoretic application of glutamate (GLU) activates DRN-5-HT neurons (VanderMaelen et al., 1986), and an autoradiographic study provided anatomical evidence for EAA projections to the DRN from several areas, with the most dense EAA projection originating in the lateral habenula (Kalen et al., 1985). In addition, lesions of the lateral habenula result in reduced high-affinity GLU uptake in the DRN, providing further evidence that the habenulo-raphe projection utilizes, at least in part, an EAA neurotransmitter (Kalen et al., 1986). Physiological evidence also supports this idea. Electrical stimulation of the lateral habenula increases 5-HT release in the striatum, and this effect is blocked by infusing the EAA antagonist kynurenic acid (KYN) into the DRN (Kalen et al., 1989).

The DRN also receives a dense noradrenergic input, as demonstrated by histofluorescence, and by biochemical, immunocytochemical, and autoradiographic methods (Fuxe, 1965; Swanson and Hartman, 1975; Anderson et al., 1977; Baraban and Aghajanian, 1981). It has further been shown by electron microscopy that noradrenergic terminals in the DRN synapse directly onto dendrites of identified 5-HT neurons (Baraban and Aghajanian, 1981). Although there is evidence for a noradrenergic projection from the locus coeruleus (LC), most of the noradrenergic input appears to arise from nuclei outside the LC (Roizen and Jacobowitz, 1976; Anderson et al., 1977). It has been suggested that the activity of 5-HT neurons is dependent on this noradrenergic innervation (Baraban and Aghajanian, 1980).

The present studies investigated the functional roles of the GABAergic, EAA, and noradrenergic inputs in regulating the tonic activity of DRN-5-HT neurons during different stages of the sleep-wake cycle, and the phasic activity evoked by sensory stimuli.

\section{Materials and Methods}

Surgical preparation. Adult male or female cats $(3.0-5.0 \mathrm{~kg})$ were anesthetized with sodium pentobarbital $(35 \mathrm{mg} / \mathrm{kg}$, i.p.) and placed in a stereotaxic instrument. Electrodes were then implanted for recording electroencephalogram (EEG), electrooculogram (EOG), and neck electromyographic activity (EMG). A small stainless steel cylinder, which could later be attached to a head-restraint apparatus, was also cemented to the animal's skull. This allowed the animal's head to be rigidly fixed in space at known stereotaxic coordinates in an atraumatic, painless manner.

Cats were treated once the night before surgery, twice on the day of surgery, and once on the morning after surgery with dexamethasone and procaine penicillin $\mathrm{G}$ (Azimycin, $0.5 \mathrm{ml}$, i.m.). Cats were then treated postoperatively, twice daily, for up to $7 \mathrm{~d}$ with Combiotic (procaine penicillin $\mathrm{G}$ and dihydrostreptomycin, $0.3 \mathrm{ml}$, i.m.) and Polyflex (ampicillin, $0.3 \mathrm{ml}$, i.m.). The implant incision was cleaned daily with hydrogen peroxide and a topical antibiotic powder was applied in order to prevent scalp infections.

After a 2 week recovery period, and $2-3 \mathrm{~d}$ before recording sessions began, a craniotomy was made in the skull. This craniotomy could not be made at the time of initial surgery since, with time, scar tissue formation and dura regrowth would form a barrier to electrode penetration. For this procedure, cats were anesthetized with the short-acting, nonbarbiturate anesthetic ketamine $\mathrm{HCl}(45 \mathrm{mg} / \mathrm{kg}, \mathrm{i} . \mathrm{m}$.$) and placed$ into the head-restraint apparatus. A small hole (2-3 mm diameter) was drilled in the skull to provide access to the DRN (P 0-2, L O, H 0). The dura was cut and carefully removed, and the hole was covered with an antibiotic cream and sealed with sterilc bonc wax. Cats were then treated with antibiotics and allowed to recover for $2-3 \mathrm{~d}$ before initiating recording sessions.
Recording/micrniontophoresis procedures. A combination recording/ microiontophoretic electrode was constructed in our laboratory by cementing a single-barrel glass pipette with a $1-2 \mu \mathrm{m}$ tip alongside a fivebarrel glass pipette with a 7-10 $\mu \mathrm{m}$ total tip diameter. The rccording barrel contained $2 \mathrm{M} \mathrm{NaCl}$ and extended approximately $20-30 \mu \mathrm{m}$ beyond the tips of the other barrels. The remaining five barrels were used for the microiontophoretic application of drugs and vehicle solutions and for current balancing. A Medical Systems Neurophore microiontophoresis system was used for ejecting drugs. Retaining currents were generally $5-10 \mathrm{nA}$. All drugs used for microiontophoresis were dissolved in deionized $\mathrm{H}_{2} \mathrm{O}$ at $\mathrm{pH} 4.0$ unless otherwise specified. These were 5-HT creatinine sulfate, $0.04 \mathrm{M}$; norepinephrine bitartrate $(\mathrm{NE}), 0.1 \mathrm{M}$; phenylephrine $\mathrm{HCl}(\mathrm{PE}), 0.1 \mathrm{M}$; L-glutamic acid, $0.2 \mathrm{M}, \mathrm{pH} 7.5 ; \mathrm{GABA}, 0.5$ $\mathrm{M}, \mathrm{pH} 3.0$; bicuculline methiodide (BIC), $0.01 \mathrm{M}$ in $165 \mathrm{mM} \mathrm{NaCl}$; and kynurenic acid, $0.1 \mathrm{M}, \mathrm{pH} 8.0$

Single-unit recordings were amplified [World Precision Instruments (WPI) electrometer and Grass P511 AC preamp], filtered (bandpass 0.3 $\mathrm{Hz}$ to $3 \mathrm{KHz}$ ), monitored continuously on an oscilloscope, and stored on an FM tape deck (Vetter model D). Single-unit activity was distinguished with a window discriminator (WPI model 121), and gated pulses were recorded on tape, polygraphically, and led to a Commodore 64 computer for off-line analysis. Gross potentials from the EEG, EOG, and EMG leads, as well as stimulus markers for the onset and offset of iontophoretic drug ejection, were also recorded on tape and polygraphically.

At the beginning of each recording session, the cat was placed in the stereotaxic head-restraint device, which holds the head in an atraumatic manner, without pressure or pain. Head movements were therefore restrained, but the trunk and limbs were free to move. Cats readily adapted to this procedure and would commonly initiate several sleepwake cycles during a typical 4-6 hr recording session. The animal was returned to its home cage at any sign of distress or discomfort. Between recording sessions, the craniotomy was covered with antibiotic cream and sealed with sterile bone wax.

Experimental protocol. Neurons were identified as serotonergic if they met the following criteria: (1) slow and highly regular activity during quiet waking $(\sim 1.0-4.0 \mathrm{~Hz}),(2)$ long-duration action potential $(>2$ msec), (3) changes in activity directly correlated with changes in behavioral state, and (4) subsequent histological localization in the DRN (Fornal and Jacobs, 1988).

After isolating the single-unit activity of a neuron that met the above criteria, the ejection currents for the agonist and antagonist were established. The agonist was first applied at regular intervals with increasingly higher ejection currents until one of the following conditions was met. For inhibitory effects, current was increased to establish an ejection current that produced complete suppression of neuronal activity within 5-10 sec of the onset of ejection. For excitatory effects, current was increased to establish an ejection current that produced a two- to threefold increase in firing rate, also within 5-10 sec of onset. Ejection of the antagonist was then paired with the corresponding agonist at the preestablished current. The antagonist was applied at varying currents to determine the threshold current necessary to either block or reverse the agonist-induced effect. Bicuculline was used to block the effects of GABA (Curtis et al., 1971), and kynurenic acid was used to block the effects of glutamate (Ganong et al., 1983; Stone et al., 1986).

The cat was then left undisturbed, and either the agonist or the antagonist was applied for 30-60 sec epochs during different stages of the sleep-wake cycle, as defined by polygraphic criteria. Mean unit activity during the drug ejection period was compared to the predrug firing rate obtaincd from a $60 \mathrm{sec}$ baseline period. Current, $\mathrm{pH}$, and vehicle control ejections were also carried out on some neurons. Sensory responses were elicited with a $100 \mathrm{~dB}$ click, produced by means of a $0.5-\mathrm{msec}$ duration square wave pulse, delivered in blocks of $20-30$ trials with a $3 \mathrm{sec}$ interstimulus interval. Unit activity and stimulus markers were recorded on FM tape, and peristimulus time histograms (PSTHs) were obtained off line using a Commodore 64 computer. For the sensory response studies, a predrug baseline response was first obtained. Ejection of the drug was then initiated, and after a $60 \mathrm{sec}$ delay, a second sensory response was obtained while drug ejection continued. Finally, a postdrug response was obtained 5 min after terminating iontophoretic application.

Data analysis. For the analysis of tonic firing rate, mean firing rates were compared using the Student's $t$ test for paired samples or repeatedmcasures analysis of variance (ANOVA). The periods used for comparing firing rates under different conditions were matched for equivalent behavioral state using polygraphic criteria. In order to quantify 
sensory-evoked responses, peristimulus time histograms (PSTHs) were computer generated off line, typically with bin widths of 10-50 msec. Mean and standard error of the mean (SEM) of counts per bin were determined for a $500 \mathrm{msec}$ prestimulus baseline period. The duration of the response was determined by the number of consecutive bins whose counts differed from baseline activity by two standard errors and were separated by no more than one nonsignificant bin.

The magnitude of the response (R-mag), was then calculated according to the following formula:

$$
\mathrm{R}-\mathrm{mag}=\text { \# of evoked spikes in excitatory period/\# trials, }
$$

where

$$
\begin{aligned}
\# \text { evoked spikes }= & \# \text { counts in excitatory period } \\
- & \text { (mean \# counts per baseline bin } \\
& \times \text { \# bins in excitatory period). }
\end{aligned}
$$

The response magnitudes for the baseline, drug, and recovery conditions were then statistically compared using a repeated-measures ANOVA.

Histology. At the completion of the study, cats were deeply anesthetized with sodium pentobarbital. A stainless steel microelectrode was then lowered to the spot where the final unit recording was obtained, and a $20 \mu \mathrm{A}$ direct anodal current was passed through the electrode for $15 \mathrm{sec}$ to deposit iron at the electrode tip. A second spot was made at a known distance from the first spot to allow adjustments for tissue shrinkage, and one or two key locations relative to other recording sites were similarly marked. The cats were then perfused intracardially with physiological saline followed by $10 \%$ formalin and then $5 \%$ potassium ferrocyanide in formalin. Brains were removed, blocked, sectioned at $50 \mu \mathrm{m}$, mounted on slides, and stained with neutral red. Recording sites (blue spots) were located, tissue shrinkage estimated, and electrode tracks reconstructed.

\section{Results}

\section{General}

The single-unit activity of 110 presumed 5-HT neurons was examined in these studies. Seventy-four of these neurons (recorded from 16 cats) were successfully studied during the microiontophoretic application of drugs, and met the following criteria for DRN-5-HT neurons: (1) a slow and regular firing rate during quiet waking (mean $\pm \mathrm{SEM}=2.53 \pm 0.16$ spikes/ sec), (2) an action potential duration of $>2 \mathrm{msec}$, (3) changes in firing rate directly related to changes in behavioral state, and (4) histological localization to the DRN (Fornal and Jacobs, 1988). In addition, 10 of these neurons (recorded from five cats) were successfully recorded during an episode of REM sleep. All 10 neurons were virtually silent during REM sleep, with a mean firing rate of $0.03 \perp 0.01$ spikes/sec, representing a highly significant decrease of over $98 \%$ from quiet waking levels $(p<$ 0.001 ). Thus, the characteristics of this neuronal population are virtually identical to those previously reported for DRN-5-HT neurons in freely moving cats (McGinty and Harper, 1976; Trulson and Jacobs, 1979).

The effect of iontophoretically applied 5-HT on neuronal activity during quiet waking was also investigated on 10 DRN-5HT neurons. Examples of the response of DRN-5-HT neurons to iontophoretic application of 5-HT are shown in Figure 1. The activity of all 10 neurons tested with $5-\mathrm{HT}$ was completely inhibited with ejection currents of 10-50 nA. Recovery to baseline levels generally occurred in $10-15 \mathrm{sec}$. These results are consistent with previously reported studies in the DRN of anesthetized rats (Aghajanian et al., 1972; Sprouse and Aghajanian, 1986), and further support the serotonergic identity of the neurons recorded in the present study.

\section{GABAergic input}

The effect of microiontophoretic application of GABA was tested on 44 DRN-5-HT neurons recorded during quiet waking.
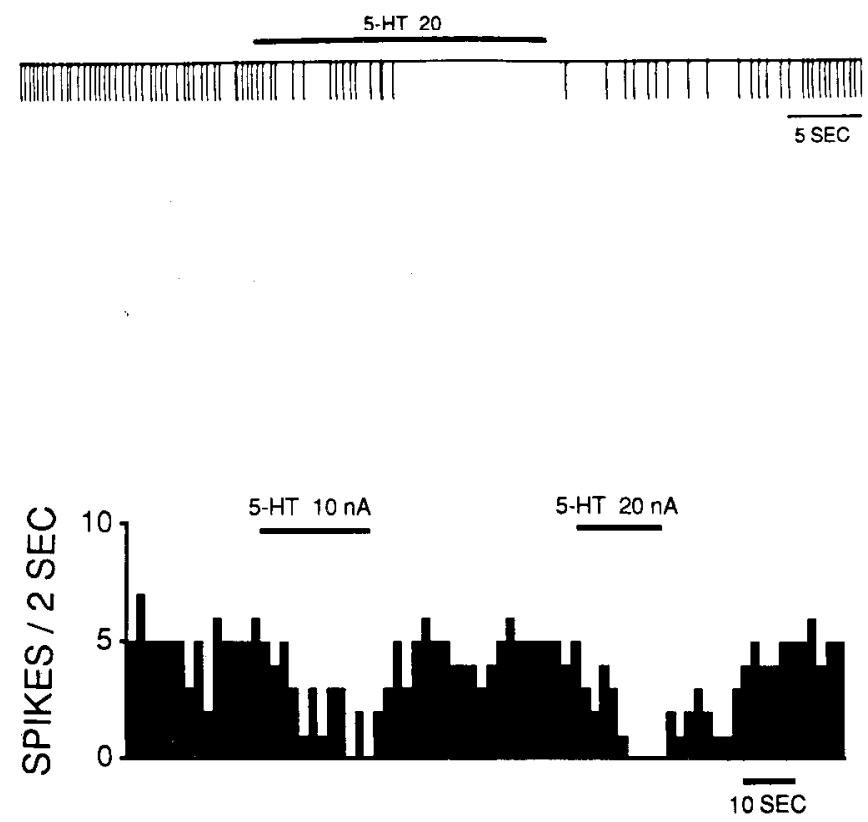

Figure 1. Examples of the neuronal response to iontophoretically applied 5-HT on DRN-5-HT neurons recorded during quiet waking. Top panel, Polygraph record of the neuronal response to 5-HT applied with $20 \mathrm{nA}$. The solid bar indicates the period of application of 5-HT. Bottom panel, Computer-generated ratemeter example of the response of another DRN-5-HT neuron to the microiontophoretic application of 5-HT Note the increased neuronal response with the higher ejection current.

These neurons were rapidly and completely inhibited by GABA, typically applied with a current of $20 \mathrm{nA}$. The $\mathrm{GABA}_{\mathrm{A}}$ receptor antagonist $\mathrm{BIC}(15-150 \mathrm{nA})$ completely reversed or blocked the GABA-induced inhibition on 32 out of 35 neurons (recorded from 11 animals). The $\mathrm{BIC}$-induced reversal for the remaining three neurons was incomplete with the ejection currents tested. An example of the neuronal response to GABA and its blockade by BIC is shown in the top panel of Figure 2 and in the left portion of Figure 3. Iontophoretic application of a $\mathrm{pH}$-matched saline solution had no effect on the GABA-mediated inhibition $(n=3)$, and BIC had no effect on the inhibition produced by iontophoresis of 5-HT ( $n=3$; right portion of Fig. 3). In addition, there was no change in the waveform characteristics of the recorded spike during iontophoresis of either GABA or BIC.

In order to investigate the functional role of the GABAergic input to the DRN, BIC was iontophoretically applied during different stages of the sleep-wake cycle. For each neuron tested, BIC was applied at a current that was effective in completely reversing or blocking the effect of iontophoretically applied GABA on that particular neuron. The effect of BIC alone on DRN-5-HT neuronal activity during quiet waking was studied on 15 neurons (recorded from seven animals). Examples of the response to iontophoretically applied BIC during quiet waking are shown in the bottom panel of Figure 2 and the top panel of Figure 4. As summarized in the left portion of Figure 5, application of BIC alone during quiet waking (15-150 nA; mean duration $=60 \mathrm{sec}$ ) had no effect on maintained activity of DRN5 -HT neurons (mean baseline firing rate $=2.72 \pm 0.22$ spikes/ sec; mean firing rate during $\mathrm{BIC}=2.41 \perp 0.17$ spikes $/ \mathrm{sec} ; p>$ $0.6)$.

In contrast to its lack of effect during quiet waking, iontophoresis of BIC during slow wave sleep (SWS) produced a significant increase in neuronal activity (the current levels used 


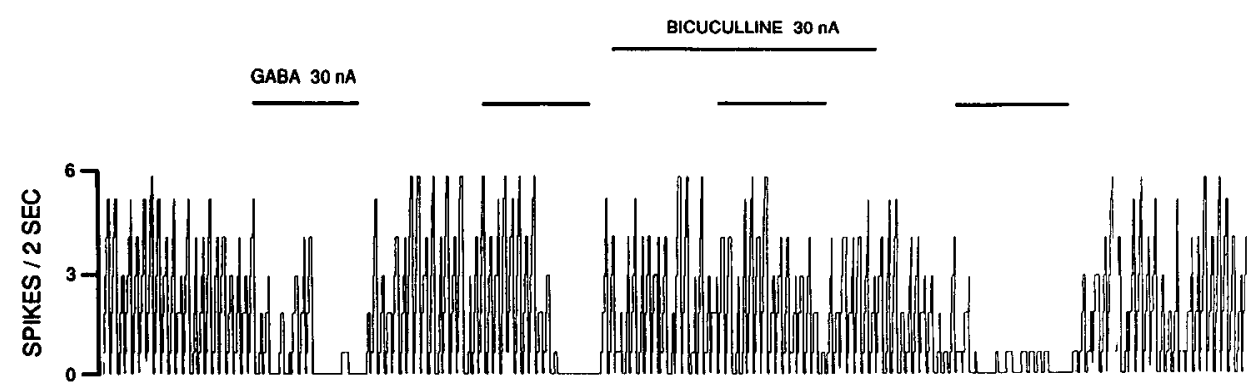

Figure 2. Ratemeter example of the neuronal response to iontophoretic application of GABA and the GABA antagonist drug BIC during quiet waking. Top panel. The activity of this neuron was completely inhibited by iontophoretic application of GABA with 30 $\mathrm{nA}$, and this suppression was blocked by application of BIC. Bottom panel, The maintained activity of this neuron during quiet waking was unaffected by application of BIC alone with $75 \mathrm{nA}$.

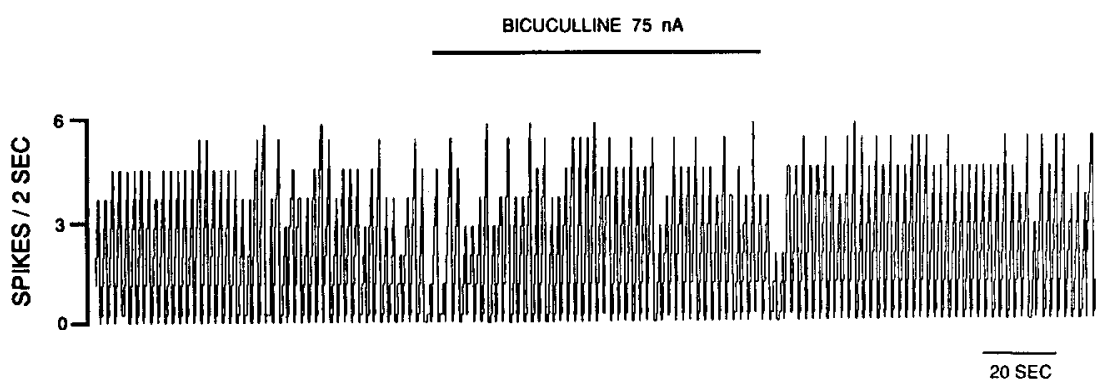

during SWS were identical to those used during quiet waking). An example of the effect of BIC during SWS for a single DRN5 -HT neuron is shown in the bottom panel of Figure 4. As summarized in Figure 5, the mean baseline firing rate during SWS was $1.49 \pm 0.38$ spikes $/ \mathrm{sec}(n=8$; three animals), while the application of BIC $(15-150 \mathrm{nA}$; mean duration = $30 \mathrm{sec})$ increased mean firing rate to $2.50 \pm 0.40$ spikes $/ \sec (p<0.01)$. All eight neurons tested showed an increase in activity during application of BIC during SWS. The mean firing rate evoked by iontophoretic application of BIC during SWS $(2.50 \pm 0.40$ spikes/sec) was not significantly different than the quiet waking baseline firing rate for these neurons $(3.04 \pm 0.42 \mathrm{spikes} / \mathrm{sec})$ $(p>0.5)$. Control ejections of a $\mathrm{pH}$-matched saline solution had no effect on the maintained firing rate of DRN-5-HT neurons during either quiet waking or SWS $(n=5 ; p>0.3)$.

The effect of iontophoretically applied BIC was also examined during REM sleep on six DRN-5-HT neurons. The mean firing rate of these neurons during REM sleep was $0.04 \pm 0.1$ spikes/ $\mathrm{sec}$. As summarized in Figure 5, the activity of these six neurons was not significantly affected by iontophoretic application of BIC applied with the same currents as used above $(p>0.8)$,



Figure 3. Computer-generated ratemeter example of the response of a DRN-5-HT neuron to the application of GABA, 5-HT, and BIC during quiet waking. On the left, iontophoretic application of BIC reversed the effect of application of GABA, but, on the right, it had no effect on the neuronal response to 5-HT application. although the activity of two of these neurons was slightly increased by BIC application during REM sleep.

\section{EAA input}

The effect of microiontophoretic application of GLU was studied on 26 DRN-5-HT neurons. All of these neurons were activated by GLU, with a significant activation often seen with currents as low as $5 \mathrm{nA}$. With high ejection currents $(>50 \mathrm{nA})$, firing rates as high as $15-20 \mathrm{spikes} / \mathrm{sec}$ were elicited. The effect of the EAA antagonist KYN was tested on seven neurons (recorded from three animals). The excitation produced by GLU was completely blocked by application of KYN (50-150 nA) in all seven neurons. The top panel of Figure 6 illustrates the response of a single DRN-5-HT neuron to iontophoretically applied GLU and the blockade of this response by iontophoresis of KYN. Saline ejection had no effect on the excitation produced by iontophoresis of GLU $(n=3)$. These seven neurons were then studied with KYN alone during quiet waking (100-300 $\mathrm{nA} ; 60 \mathrm{sec}$ duration). The bottom panel of Figure 6 shows an example of the neuronal response to iontophoresis of KYN. Mean firing rate was not significantly affected by KYN alone during quiet waking (mean baseline firing rate $=1.82 \pm 0.24$ spikes/sec; mean firing rate during $\mathrm{KYN}=1.75 \pm 0.24$ spikes/ $\sec ; p>0.1)$.

The activity of the same seven neurons was also studied in response to the presentation of a series of 20-30 clicks. PSTHs for the response of a single DRN-5-HT neuron are shown in Figure 7. The top panel shows the baseline evoked response for this neuron. As shown in the middle panel, the phasic sensory response of this neuron was almost completely abolished during iontophoresis of KYN (200 nA), and the response showed completely recovery $5 \mathrm{~min}$ after the iontophoretic application was terminated (bottom panel). The mean group data for the auditory evoked responses of these neurons are summarized in the top panel of Figure 8. Under baseline conditions, the mean response magnitude was $0.50 \pm 0.05$ evoked spikes/trial. This response was significantly reduced during iontophoresis of KYN $(100-200 \mathrm{nA})$ to $0.16 \pm 0.05$ evoked spikes/trial $(p<0.001)$. 


\section{QUIET WAKING}

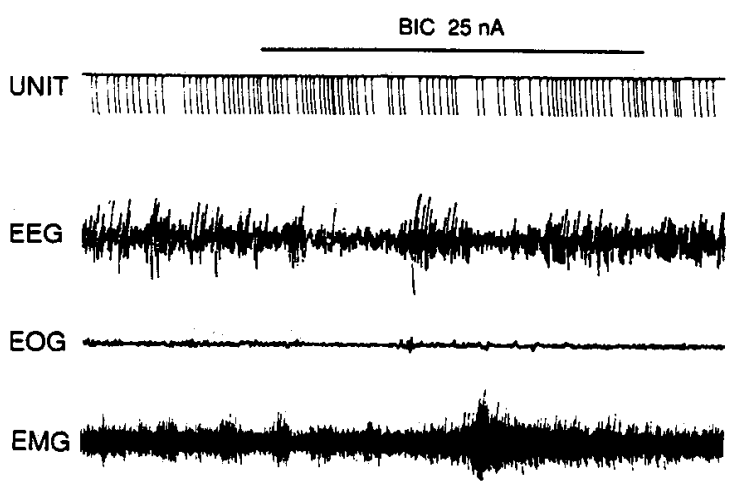

SLOW WAVE SLEEP

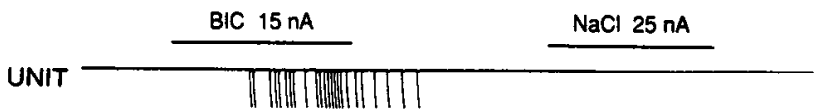

EEG

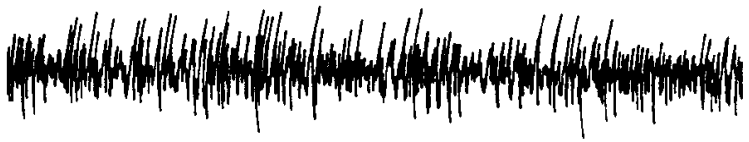

EOG

EMG

Figure 4. Polygraph records displaying the activity of a single DRN5-HT neuron and gross potentials in response to the iontophoretic application of the GABA antagonist BIC. Top panel, Effect of iontophoretic application of BIC during quiet waking. Bottom panel, Response of the same neuron to iontophoretic application of BIC and saline during SWS (this particular neuron showed no spontaneous activity during this stage of SWS). Short-duration applications are shown here for illustrative purposes (typical durations were $30-60 \mathrm{sec}$ ).

Five minutes after terminating the iontophoresis of KYN, the evoked response showed a partial and significant recovery, with a mean response magnitude of $0.34 \pm 0.07$ spikes/trial $(p<$ $0.01)$.

It is important to note that while the evoked response of DRN5-HT neurons was significantly decreased during iontophoresis of KYN, prestimulus maintained activity was unchanged during this time, as summarized in the bottom panel of Figure 8 (mean baseline prestimulus activity $=0.68 \pm 0.1$ spikes $/$ trial, mean prestimulus activity during $\mathrm{KYN}=0.65 \pm 0.1$ spikes/trial; $p$ $>0.8$ ). In addition, as shown in Figure 9, iontophoretic application of a $\mathrm{pH}$-matched saline solution had no significant effect on either the evoked response to auditory stimuli $(n=5 ; p>$ $0.6)$ or prestimulus maintained activity $(n=5 ; p>0.3)$. Finally, there was no change in either the duration or amplitude of the recorded spike during iontophoresis of either GLU or KYN.

\section{Noradrenergic input}

In the present studies, the activity of eight DRN-5-HT neurons was recorded during the iontophoretic application of either NE

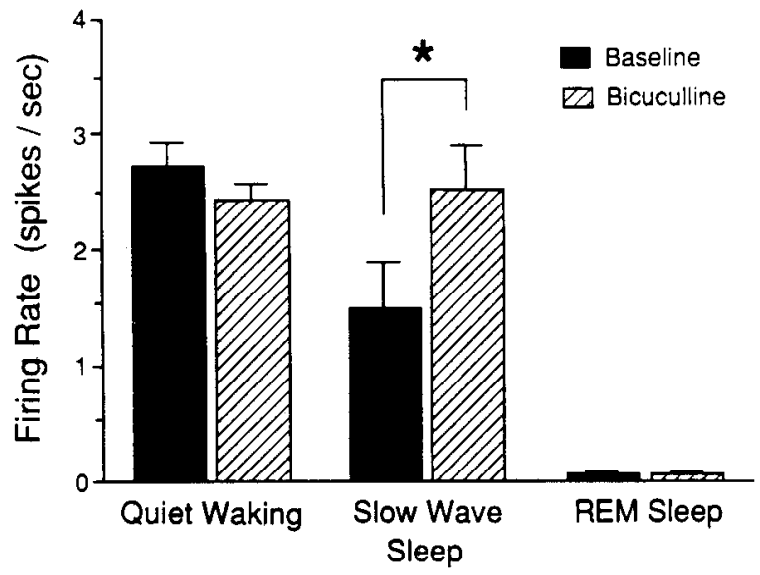

Figure 5. Effect of iontophoretic application of the GABA antagonist BIC on DRN-5-HT ncuronal activity across the sleep-wake cycle. BIC was applied for 30-60 sec with a preestablished current (range, 15-150 $\mathrm{nA}$ ). Neuronal activity during SWS was significantly increased by iontophoretic application of $\operatorname{BIC}(n=8 ; *, p<0.01 ;$ middle panel $)$, whereas iontophoretic application of BIC did not significantly affect activity in either quiet waking $(n=15 ; p>0.6$; left panel) or REM sleep $(n=6$; $p>0.8 ;$ right panel). It is interesting to note that the firing rate evoked by iontophoresis of BIC during SWS was not significantly different than the quiet waking baseline activity of these eight neurons $(p>0.5)$. Values are mean + SEM.

or the specific $\alpha$-adrenergic agonist drug PE. The effect of iontophoretic application of $\mathrm{NE}$ during quiet waking was examined on three neurons (recorded from two animals). NE (25-50 nA; $30-60 \mathrm{sec}$ ) had no effect on maintained activity of these neurons during quiet waking (mean baseline firing rate $=2.67 \pm 1.1$ spikes/sec; mean firing rate during $\mathrm{NE}=2.62 \pm 1.2 \mathrm{spikes} / \mathrm{sec}$; $p>0.7)$. Since previous studies have reported that the effects of NE on DRN-5-HT neuronal activity are mediated by an $\alpha$-adrenergic receptor (Gallager and Aghajanian, 1976a; Baraban and Aghajanian, 1980), the activity of five other neurons (recorded from three animals) was studied during iontophoretic application of PE, a selective $\alpha_{1}$-receptor agonist. PE (25-50 nA;
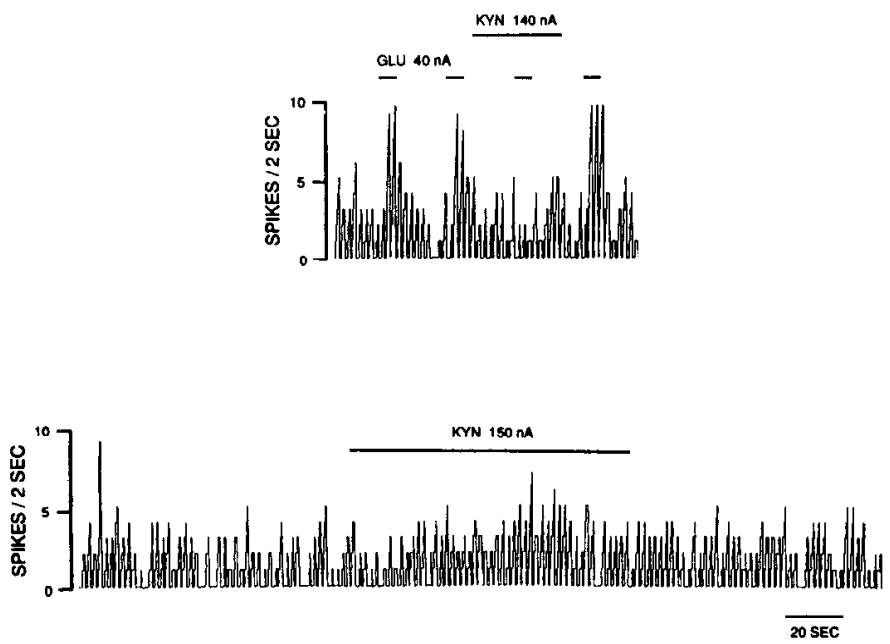

Figure 6. Ratemeter example of the response of a single DRN-5-HT neuron to iontophoretic application of GLU and the EAA antagonist KYN. Top panel, Iontophoresis of KYN $(140 \mathrm{nA})$ blocked the excitation produced by iontophoresis of GLU (40 nA). Bottom panel, The spontaneous activity of the same neuron during quiet waking was unaffected by iontophoresis of KYN alone. 

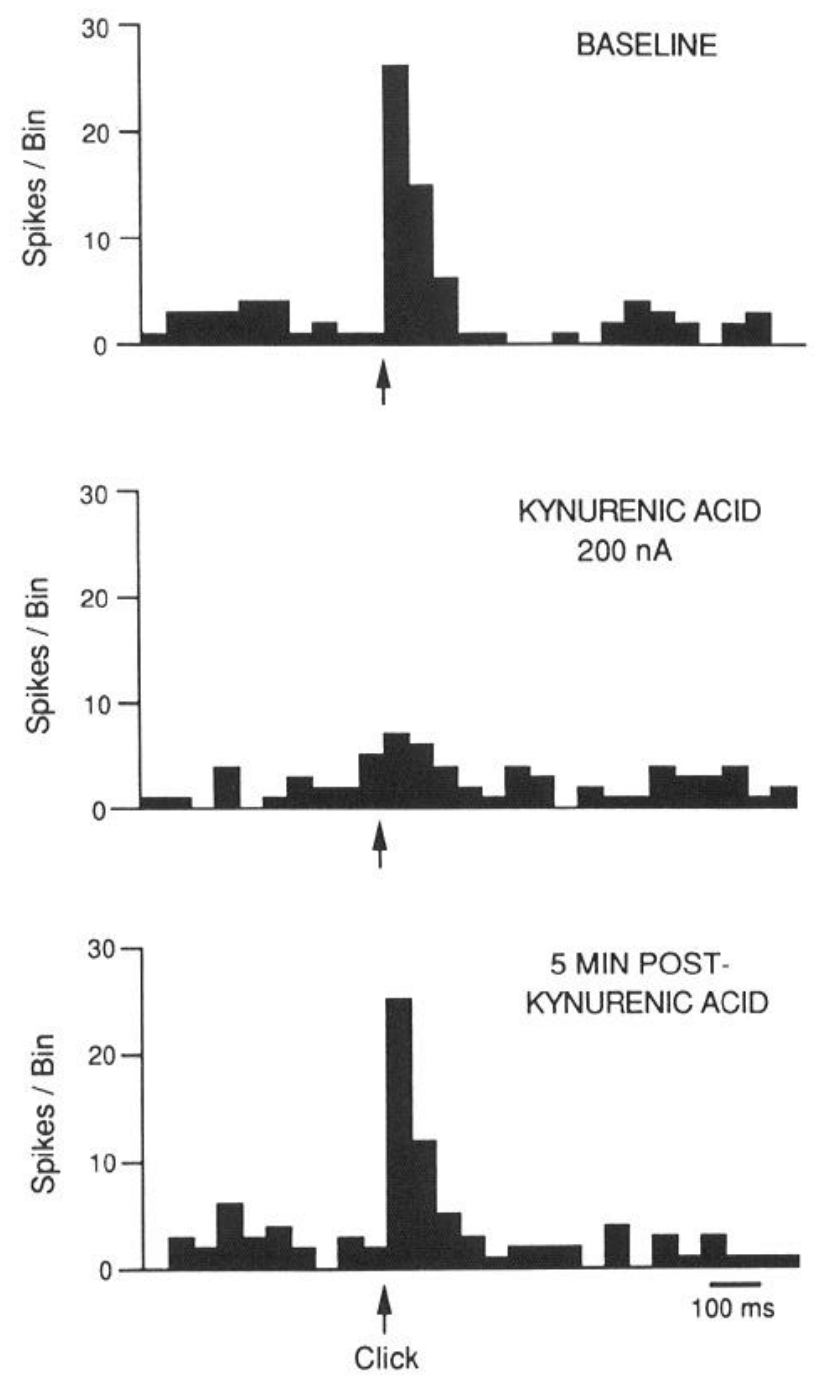

Figure 7. Computer-generated PSTHs of the response of a DRN-5HT neuron to the presentation of a series of auditory clicks. Top panel shows the neuronal response under baseline conditions. The evoked response was attenuated during the microiontophoresis of the EAA antagonist KYN (middle panel), and recovered $5 \mathrm{~min}$ after termination of iontophoretic current (bottom panel). These histograms represent the summation of 60 presentations of the stimulus occurring once every 2 sec. Bin width $=50 \mathrm{msec}$.

30-60 sec) also had no significant effect on firing rate during quiet waking (mean baseline firing rate $=3.06 \pm 0.5$ spikes $/ \mathrm{sec}$; mean firing rate during $\mathrm{PE}=2.93 \pm 0.5$ spikes $/ \mathrm{sec} ; p>0.1$ ).

It should be noted that NE and PE were only applied to neurons that were activated by iontophoretic ejection of GLU from an adjacent iontophoretic barrel. This was to ensure that the observed lack of effect of NE and PE was not due to a problem with the electrode (e.g., situated too far from the cell body or separation between drug and recording barrels). In addition, consistent with previous studies (Gallager and Aghajanian, 1976a; Baraban and Aghajanian, 1980), inhibition of firing was occasionally produced by high ejection currents of NE or $\mathrm{PE}(>50 \mathrm{nA})$, indicating that the drugs were being successfully ejected from the iontophoretic barrels.

\section{Discussion}

As reviewed above, DRN-5-HT neurons receive numerous anatomical inputs utilizing a diversity of neurotransmitters (Jacobs


Figure 8. Effect of the microiontophoretic application of the EAA antagonist KYN on the DRN-5-HT neuronal response to auditory stimuli. Top panel, Microiontophoresis of KYN (100-200 nA) significantly reduced the evoked response to auditory stimuli $\left(n=7 ;{ }^{*}, p<0.001\right)$. The response showed significant recovery $5 \mathrm{~min}$ after termination of the iontophoresis of KYN $(n=6 ; * *, p<0.01)$. Bottom panel, Prestimulus maintained activity was unaffected by the iontophoretic application of $\mathrm{KYN}(n=7 ; p>0.8)$. Values are mean \pm SEM.

and Azmitia, 1992). The present studies were designed to investigate the neurochemical afferents that regulate the activity of DRN-5-HT neurons under specific physiological conditions. This was addressed by using microiontophoretic application of neurotransmitters and their antagonists in the awake cat. By conducting these studies in the awake animal and using antagonists, it was possible to inactivate specific neurochemical inputs selectively and reversibly during the conditions that normally influence DRN-5-HT neuronal activity. A similar approach has been successfully used to study the neurochemical modulation of the response properties of neurons in other brain areas in awake animals (Sawaguchi, 1987; Chase et al., 1989). Thus, by capitalizing on the known anatomy and electrophysiology of DRN-5-HT neurons, the present studies were able to test specific hypotheses regarding the afferent control of DRN-5-HT neuronal activity under physiological conditions.

In order to investigate the functional role of the GABAergic input to the DRN, the GABA antagonist BIC was iontophoretically applied during different stages of the sleep-wake cycle. The effects of BIC on DRN-5-HT neuronal activity were state dependent, suggesting that the endogenous GABAergic input to these neurons is involved in regulating their state-dependent activity. While blockade of GABA receptors had no effect on the maintained activity of DRN-5-HT neurons during quiet waking, the firing rate of these neurons during SWS was significantly increased by GABA receptor blockade. This suggests that a GABAergic input is specifically involved in mediating the suppression of activity of DRN-5-HT neurons during SWS. The site of origin of this state-dependent GABAergic input to 
the DRN is not known. The pontine reticular formation may provide a GABAergic input to the DRN (Wang et al., 1976), and there are neurons located in this area that increase their firing rate during sleep (Siegel and Rogawski, 1988). There may also be GABAergic interneurons within the DRN (Belin et al., 1979), and a GABA-mediated suppression of neuronal activity could be produced by an excitatory input to GABAergic interneurons that in turn project to DRN-5-HT neurons.

During REM sleep, DRN-5-HT neurons are virtually silent, and iontophoretic application of BIC was generally ineffective in reversing this suppression of activity. It may be that the GABAergic input to the DRN is intensified during REM sleep, and iontophoretic application of BIC with the currents used was insufficient to reverse this increased inhibitory drive. There may also be other inputs, such as a cholinergic input (Gallardo and Pasquier, 1980) or a glycine input (Snyder, 1975), that participate in the suppression of neuronal activity during REM slccp.

There is also evidence for a major EAA input to the DRN (Kalen et al., 1985, 1986), but the functional role of this input is unclear. In the present studies, the EAA antagonist KYN was used to investigate the role of this input in regulating the activity of DRN-5-HT neurons. Iontophoretic application of KYN did not alter the spontaneous firing rate of DRN-5-HT neurons during quiet waking. KYN did, however, significantly reduce the magnitude of the excitatory response to phasic auditory stimuli, suggesting that an EAA input is involved in mediating this response. The lack of effect of KYN on prestimulus maintained activity rules out a direct inhibitory effect of KYN, and suggests that the maintained activity of these neurons is not dependent on this input.

Previous studies have also identified a dense noradrenergic input to the DRN (Fuxe, 1965; Swanson and Hartman, 1975), but the effect of NE on DRN-5-HT neuronal activity is unclcar, as is the functional role of this input. In the present studies, iontophoretic application of NE or the selective $\alpha_{1}$-agonist PE did not alter the firing rate of these neurons during wakefulness. This may indicate that the endogenous noradrenergic input to the DRN is maximal during wakefulness, precluding an effect with exogenously applied NE. Previous studies conducted in anesthetized animals or in vitro preparations, however, have in some cases reported facilitation of DRN-5-HT neuronal activity with iontophoresis of NE (Baraban et al., 1978; VanderMaelen and Aghajanian, 1983), possibly reflecting a diminished endogenous noradrenergic input present in these preparations.

It has also been shown that iontophoretic and systemic administration of noradrenergic antagonists suppresses DRN-5HT neuronal activity in the anesthetized animal, suggesting the presence of a tonic facilitatory input (Baraban and Aghajanian, 1980). Since the noradrenergic input would be diminished during sleep (Jacobs, 1986), a noradrenergic disfacilitation may contribute to the suppression of DRN-5-HT neuronal activity during sleep. Systemic administration of these antagonists in the awake animal, however, has minimal effects on neuronal activity (Heym et al., 1981). Additional studies examining the effect of iontophoretically applied noradrenergic antagonists in the awake animal, as well as the effect of NE applied to these neurons during sleep, may shed more light on the functional role of the noradrenergic input to the DRN.

The present results may also facilitate the study of the functional roles of DRN-5-HT neurons in various physiological and behavioral processes. If distinct neurochemical inputs regulate specific aspects of DRN-5-HT neuronal activity, it may be pos-
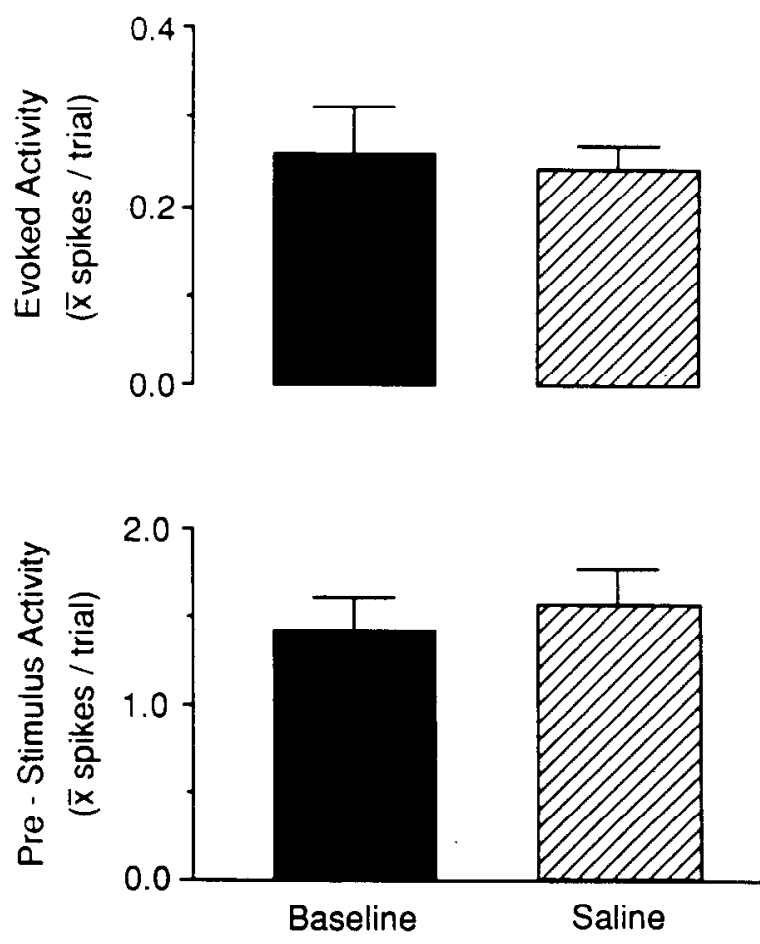

Figure 9. Effect of the microiontophoretic application of saline on the DRN-5-HT neuronal response to auditory stimuli. Top panel, Iontophoresis of saline (100-200 nA) had no significant effect on evoked activity $(n=5 ; p>0.6)$. Bottom panel, Prestimulus maintained activity was also unaffected by iontophoresis of saline $(p>0.3)$. Values are mean $\pm \mathrm{SEM}$.

sible to disrupt these neuronal responses selectively to investigate their role in the relevant behavior. For example, if the EAA input to the DRN specifically mediates the neuronal response to phasic sensory stimuli, then local infusion of an EAA antagonist into the DRN might selectively block the sensory response of these neurons without affecting their tonic maintained activity. This would allow for an investigation of the role of this neuronal response on relevant parameters of the response to sensory stimuli, such as threshold detectability or sensitivity, at either a behavioral or a neural systems level.

In sum, the present studies demonstrate the utility of microiontophoresis in the awake animal in delineating the functional roles of specific neurotransmitter afferents to the DRN. This information is important for understanding the circuitry that regulates the activity of these neurons under physiological conditions and, ultimately, the role of the DRN-5-HT system in behavioral and physiological processes.

\section{References}

Aghajanian GK, Wang RY (1977) Habenular and other midbrain raphe afferents demonstrated by a modified retrograde tracing technique. Brain Res 122:229-242.

Aghajanian GK, Haigler HJ, Bloom FE (1972) Lysergic acid diethylamide and serotonin: direct actions on serotonin-containing neurons. Life Sci 11:615-622.

Anderson CD, Pasquier DA, Forbes WB, Morgane PJ (1977) Locus coeruleus-to-dorsal raphe input examined by electrophysiological and morphological methods. Brain Res Bull 2:209-221.

Baraban JM, Aghajanian GK (1980) Suppression of firing activity of 5-HT neurons in the dorsal raphe by alpha-adrenoceptor antagonists. Neuropharmacology 19:355-363.

Baraban JM, Aghajanian GK (1981) Noradrenergic innervation of 
serotonergic neurons in the dorsal raphe: demonstration by electron microscopic autoradiography. Brain Res 204:1-11.

Baraban JM, Wang RY, Aghajanian GK (1978) Reserpine suppression of dorsal raphe neuronal firing: mediation by adrenergic system. Eur J Pharmacol 52:27-36.

Belin MF, Aguera M, Tappaz M, McRae-Degueurce A, Bobillier P, Pujol JF (1979) GABA-accumulating neurons in the nucleus raphe dorsalis and periaqueductal gray in the rat: a biochemical and radioautographic study. Brain Res 170:279-297.

Chase MII, Soja PJ, Morales FR (1989) Evidence that glycine mediates the postsynaptic potentials that inhibit lumbar motoneurons during the atonia of active sleep. J Neurosci 9:743-751.

Curtis DR, Duggan AW, Felix D, Johnston GAK (1971) Bicuculline, an antagonist of GABA and synaptic inhibition in the spinal cord of the cat. Brain Res 32:69-96.

Fornal CA, Jacobs BL (1988) Physiological and behavioral correlates of serotonergic single unit activity. In: Neuronal serotonin (Osborne NN, Hamon M, eds), pp 305-345. New York: Wiley.

Fuxe K (1965) Evidence for the existence of monoamine-containing neurons in the central nervous system. IV. The distribution of monoamine terminals in the central nervous system. Acta Physiol Scand 64[Suppl 247]:41-85.

Gallager DW, Aghajanian GK (1976a) Effect of antipsychotic drugs on the firing of dorsal raphe cells. I. Rule of adrenergic system. Eur J Pharmacol 39:341-355.

Gallager DW, Aghajanian GK (1976b) Effect of antipsychotic drugs on the firing of dorsal raphe cells. II. Reversal by picrotoxin. Eur J Pharmacol 39:357-364.

Gallardo MRGP, Pasquier DA (1980) Increase in activity of choline acetyltransferase in the dorsal raphe nucleus following habenular deafferentation. Brain Res 194:578-582.

Ganong AH, Lanthorn TH, Cotman CW (1983) Kynurenic acid inhibits synaptic and acidic amino acid-induced responses in the rat hippocampus and spinal cord. Brain Res 273:170-174.

Gottesfeld Z, Hoover DB, Muth EA, Jacobowitz DM (1978) Lack of biochemical evidence for a direct habenulo-raphe GABAergic pathway. Brain Res 141:353-356.

Heym J, Trulson ME, Jacobs BL (1981) Effects of adrenergic drugs on raphe unit activity in freely moving cats. Eur J Pharmacol 74:117125.

Heym J, Trulson ME, Jacobs BL (1982) Raphe unit activity in freely moving cats: effects of phasic auditory and visual stimuli. Brain Res 232:29-39.

Jacobs BL (1986) Single unit activity of locus coeruleus neurons in behaving animals. Prog Neurobiol 27:183-194.

Jacobs BL, Azmitia FC (1992) Structure and function of the brain serotonin system. Physiol Rev 72:165-229.

Kalen P, Karlson M, Wiklund L (1985) Possible excitatory amino acid afferents to nucleus raphe dorsalis of the rat investigated with retrograde wheat germ agglutinin and $\mathrm{D}-\left[{ }^{3} \mathrm{H}\right]$ aspartate tracing. Brain Res 360:285-297.

Kalen P, Pritzel M, Nieoullon A, Wiklund L (1986) Further evidence for excitatory amino acid transmission in the lateral habenula projection to the rostral raphe nuclei: lesion-induced decrease of high affinity glutamate uptake. Neurosci Lett 68:35-40.

Kalen P, Strecker RE, Rosengren E, Bjorklund A (1989) Regulation of striatal serotonin release by the lateral habenula-dorsal raphe pathway in the rat as demonstrated by in vivo microdialysis: role of excitatory amino acids and GABA. Brain Res 492:187-202.

Lakoski JM, Aghajanian GK (1983) Effects of histamine, H1- and $\mathrm{H} 2$-receptor antagonists on the activity of serotonergic neurons in the dorsal raphe nucleus. J Pharmacol Exp Ther 227:517-523.
McGinty DJ, Harper RM (1976) Dorsal raphe neurons: depression of firing during sleep in cats. Brain Res 101:569-575.

Nishikawa T, Scatton B (1982) Evidence for a GABAergic inhibitory influence on serotonergic neurons originating from the dorsal raphe. Brain Res 279:325-329.

Roizen MF, Jacobowitz DM (1976) Studies on the origin of innervation of the noradrenergic area bordering on the nucleus raphe dorsalis. Brain Res 101:561-568.

Sakai K, Salvert D, Touret M, Jouvet M (1977) Afferent connections of the nucleus raphe dorsalis in the cat as visualized by the horseradish peroxidase technique. Brain Res 137:11-35.

Sawaguchi T (1987) Catecholamine sensitivities of neurons related to a visual reaction time task in the monkey prefrontal cortex. J Neurophysiol 58:1100-1122.

Scatton B, Serrano A, Rivot JP, Nishikawa T (1984) Inhibitory GA$B A e r g i c$ influence on striatal serotonergic transmission exerted in the dorsal raphe as revealed by in vivo voltammetry. Brain Res 305:343352.

Shima K, Nakahama H, Yamamoto M (1986) Firing properties of two types of nucleus raphe dorsalis neurons during the sleep-waking cycle and their responses to sensory stimuli. Brain Res 399:317-326.

Siegel JM, Rogawski MA (1988) A function for REM sleep: regulation of noradrenergic receptor sensitivity. Brain Res Rev 13:213-233.

Snyder SH (1975) The glycine synaptic receptor in the mammalian central nervous system. Br J Pharmacol 53:473-484.

Sprouse JS, Aghajanian GK (1986) (-)-Propanolol blocks the inhibition of serotonergic dorsal raphe cell firing by 5-HT la selective antagonists. Eur J Pharmacol 128:295-298.

Stern WC, Johnson A, Bronzinos ID, Morgane PJ (1981) Neuropharmacology of the afferent projections from the lateral habenula and substantia nigra to the anterior raphe in the rat. Neuropharmacology 20:979-989.

Stone TW, Connick JH, Addae JI, Smith DAS, Brooks PA (1986) The neuropharmacology of quinolinic acid and kynurenines. In: Excitatory amino acids (Roberts PJ, Storm-Mathisen J, Bradford HF, eds), pp 367-380. London: Macmillan.

Swanson LW, Hartman BK (1975) The central adrenergic system. An immunofluorescence study of the location of cell bodies and their efferent connections in the rat utilizing dopamine B-hydroxylase as a marker. J Comp Neurol 163:467-506.

Trulson ME, Jacobs BL (1979) Raphe unit activity in freely moving cats: correlation with level of behavioral arousal. Brain Res 163:135150.

VanderMaelen CP, Aghajanian GK (1983) Electrophysiological and pharmacological characterization of serotonergic dorsal raphe neurons recorded extracellularly and intracellularly in rat brain slices. Brain Res 289:109-119.

VanderMaelen CP, Matheson GK, Wilderman RC, Patterson LA (1986) Inhibition of serotonergic dorsal raphe neurons by systemic and iontophoretic administration of buspirone, a non-benzodiazepine anxiolytic drug. Eur J Pharmacol 129:123-130.

Vincent SR, Staines WA, McGeer EG, Fibiger HC (1980) Transmitters contained in the efferents of the habenula. Brain Res 195:479-484.

Wang RY, Aghajanian GK (1977) Physiological evidence for habenula as major link between forebrain and midbrain raphe. Science 197: 89-91.

Wang RY, Gallager DW, Aghajanian GK (1976) Stimulation of pontine reticular formation suppresses firing of serotonergic neurones in the dorsal raphe. Nature 264:365-367.

Whitaker-Azmitia PM, Peroutka SJ, eds (1990) The neuropharmacology of serotonin. New York: NY Academy of Science. 